\title{
A Comparative Study of Anopheles Gambiae Male Mosquito's Response to Frequency Modulated [FM] and Pulse Modulated [PM] Waves at Different Acoustic Frequencies and Distances
}

\author{
Maweu $^{1)}$ O. M, Deng ${ }^{2)}$ A. L. and Muia ${ }^{1)}$ L. M \\ ${ }^{1)}$ Physics and ${ }^{2)}$ Zoology Departments \\ Egerton University, P.O. Box 536, Egerton, Kenya \\ e-mail: mawmant@yahoo.com
}

\begin{abstract}
The efficiency of an electronic device meant to disrupt acoustic communication between male and female Anopheles gambiae mosquitoes was tested by recording approaches of the males towards the device as a function of distance and frequency. Different bioassays were conducted with broadcasts from the device and taped mimics of the female species wing beats at different distances and frequencies. A large number of the males, 85\% remained near the broadcast speaker for a significantly longer period when the broadcast sounds of the device were at $380 \mathrm{~Hz}$ than at any other frequency range. The taped mimic sounds of the female mosquitoes in flight elicited a maximum response rate of $75 \%$.

A test involving a choice between the device and taped sound mimics by male mosquitoes revealed that a high number (75\%) of them flew towards the device while $10 \%$ were attracted by the taped sounds whereas $15 \%$ were undecided and made oscillatory motion between the two sources. Additional bioassays conducted to examine the effect of the device on non-flying males showed that almost $75 \%$ were disturbed and approached the speaker within a distance of $0.5 \mathrm{~m}$, which confirmed a radiative near-field communication for these insects.
\end{abstract}

Keywords: Pulse width modulator (PWM), Bioassay, Broadcasting transmitter, Anopheles gambiae, Acoustic communication.

\section{Introduction}

Female mosquitoes in-flight produce a familiar whining sound, whose frequency reflects the wing beat of the species ${ }^{1)}$. This sound is produced when the insects are in search of proteins needed for ovulation. Bioacoustics gives the range of the frequencies generated by all species of mosquitoes to be between 150 and $500 \mathrm{~Hz}$ with maximum intensity at $380 \mathrm{~Hz}$ for Anopheles gambiae ${ }^{2)}$. The sound is transmitted in air as frequency modulated wave $(\mathrm{FM})^{1)}$ and it activates the antenna of conspecific male and provides directional indicators, which the massive sense organs, the Johnstone's organ at the base of the antenna can resolve $^{3)}$. Most insects responds to ultra violet radiations at wavelengths around 350nm and little is known of their response to longer wavelengths in the electromagnetic spectrum ${ }^{4}$.

The aim of this study is to test and analyze the performance and efficiency of an electronic device developed to disrupt acoustic communication between male and female Anopheles gambiae mosquitoes and compare that with those from FM waves emitted by female mosquitoes in flight. A male mosquito may be viewed as an electrically small antenna, whose dimensions are smaller than the wavelengths of the electromagnetic fields they radiate ${ }^{5}$.

On the other hand, in a pulse modulation system, sampling the wave at regular intervals produces a signal waveform. The sampling of the information is done by use of a pulse, and varying one of its basic characteristics such as amplitude, width (duration) or the position in accordance with the modulating signal ${ }^{6)}$. The sampled pulses bearing the characteristics of the modulating signal are impressed upon the carrier frequency and these short bursts of the modulated carrier are transmitted.

This mode of communication has several advantages compared to FM communication which include the employment of amplitude limiting techniques to minimize noise effects, transmission is made independent of fading and other difficulties and since energy is radiated only in short bursts power consumption is low.

In general, the rate of response and the distance over which the broadcast calls influenced the male's behavior increased with the frequency ${ }^{7}$. This observation made us to increase the frequency beyond $600 \mathrm{~Hz}$ and noted the response in the spectrum, ranging from 250 to $630 \mathrm{~Hz}$.

\section{Experimental}

\subsection{Generation of the pulse modulated wave [PWM]}

A frequency-modulated wave similar to the one emitted by a female mosquito in flight, that is, a carrier of $500 \mathrm{~Hz}$, modulating signal of $100 \mathrm{~Hz}$, amplitude of 5 volts and a modulation index of $2.9^{8)}$ was generated using a signal generator. The modulated wave was then passed through a ratio detector to separate the carrier from the audio. The audio was superimposed with a saw tooth wave generated by an emitter-coupled multi-vibrator and a 
pulse width wave, whose frequency could be varied, was, thus generated. This wave was transmitted to a group of males and their response noted.

\subsection{Bioassays}

\section{Insects}

Male mosquitoes, of 1 to 2 days after emergence were obtained from Kenya Pyrethrum board colony. Sexually mature males were used for different bioassays.

\subsection{Effect of PWM wave on male Anopheles gambiae mosquitoes}

A mosquito net in cuboid form measuring 40cm width and of varying lengths was used to carry out the study. A pulse width modulated wave (PWM) was introduced at one end of the net via a broadcasting speaker. Male mosquitoes in batches of twenty were introduced from the opposite end. The releasing points were varied, starting from a distance of 2 meters between the target and the releasing point at intervals of $0.5 \mathrm{~m}$ to a minimum distance of $0.5 \mathrm{~m}$. The approach of the insects towards the target was noted for each distance at a fixed frequency and amplitude. The frequency was varied and the approach was again noted for various distances. Twenty replicates were tested under each of the above conditions and a mean response calculated. The effect of the device on mosquito's behaviour was scored as follows;

1. Contacts -Number of mosquitoes flying direct to the target, that is, (speaker) and staying for more than ten seconds.

2. Approaches - Number approaching the device within a distance of $0.1 \mathrm{~m}$ and remaining within the vicinity for more than ten seconds.

3. Non- approach - Number that did not respond at all and never made any approach to the target.

4. The efficiency of the device: This was assessed by taking the sum of number making contacts (1) and the number approaching within $0.1 \mathrm{~m}$ distance (2).

\subsection{Effect of FM waves on male Anopheles gambiae mosquitoes}

A source containing taped mimic sounds of female mosquitoes in flight replaced the PWM source. The efficiency of this natural source was determined by varying the distance between the source and the target and a score was made as above.

\subsection{Effect of the PWM and FM broadcast in a choice test on the male mosquitoes}

A speaker emitting taped mosquito sound was placed on one side of the net and the PWM transmitter was placed on the opposite side (Figure 1). The distance between the two sources was 4 meters. Males were released at an equi-distance from the two sources. Both sources were switched on and by varying the distances of the two sources, responses were simultaneously noted with respect to the sources.

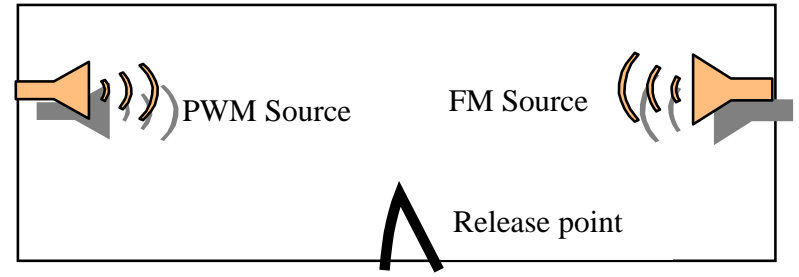

Figure 1. Experimental set up

\subsection{Effect of PWM and FM broadcasts on non- flying male mosquitoes}

In a preliminary search for measurable behaviour response to PWM broadcast, male Anopheles gambiae mosquitoes were observed for twenty minutes inside the net after the broadcast was switched off. They settled in different parts of the net with some remaining within the vicinity of the speaker. The PWM source was then switched on and the mosquito response noted and scored. The same was done with the taped female sounds.

\section{Results}

\subsection{Effect of PWM on the male's response as a function of frequency at the optimum distance.}

Figure2 shows the effect of the PWM on the male mosquito as a function of frequency at the optimum distance of $0.5 \mathrm{~m}$. It is evident that at a frequency of $380 \mathrm{~Hz}$, males were strongly attracted towards the target than at any other frequency tested at the same distance. This makes the frequency of 380 $\mathrm{Hz}$, the optimum frequency, which is one of the resonant frequencies used by these insects for the reception of acoustic waves. All tested frequencies above and below $380 \mathrm{~Hz}$ were found to attract a similar number of males, for example frequencies of $350 \mathrm{~Hz}$ and $450 \mathrm{H}$, each had a mean value of $55 \%$ response.

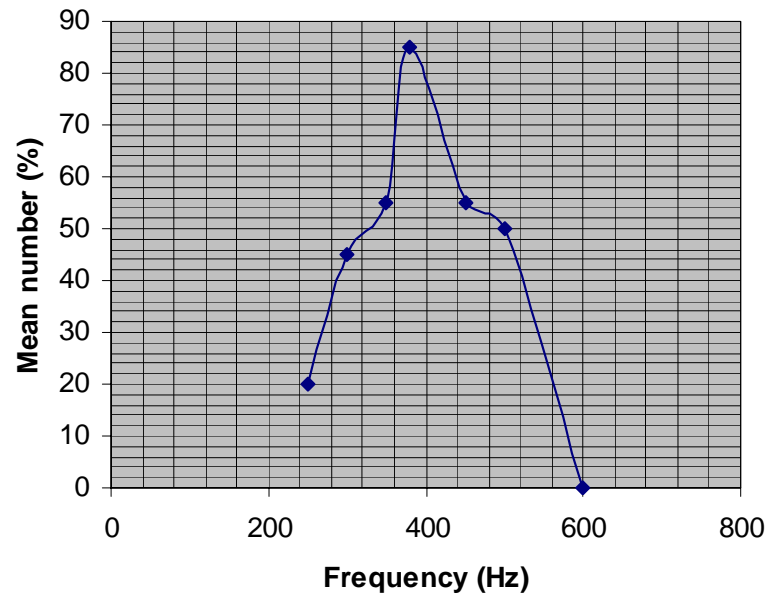

Figure 2. Male response to PWM as function of frequency at optimum distance $(0.5 \mathrm{~m})$ 


\subsection{Effect of PWM on Male's response as a function of distance at optimum frequency}

Figure3. shows the effect of PWM on the males as a function of the distance at the optimum frequency. Males were attracted more towards the target at a distance of $0.5 \mathrm{~m}$. than at any other distance at the testing frequency (optimum frequency, $380 \mathrm{~Hz}$ ). There were differences among distances with regard to mosquito attraction at optimum frequency. From the graph, it is evident that the number attracted by the target decreases exponentially with distance.

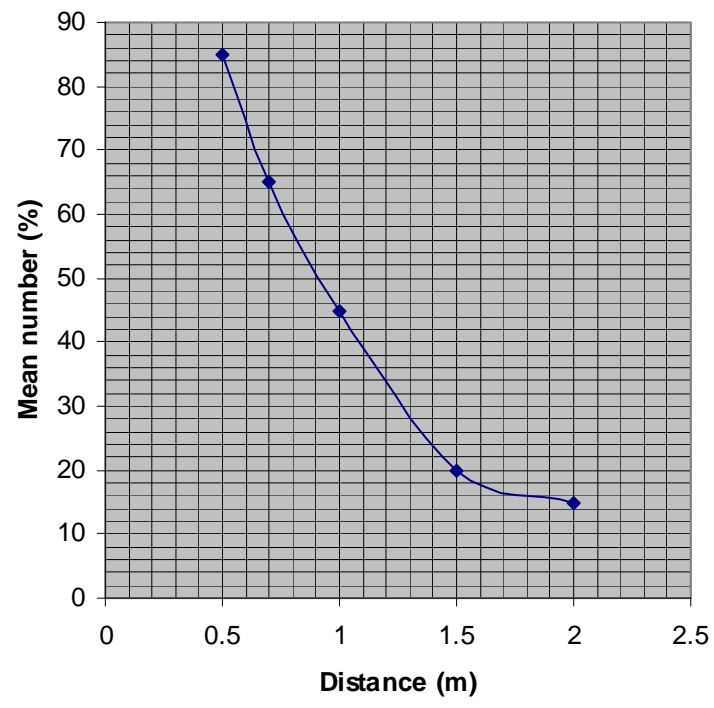

Figure 3. Males response to PWM as a function of distance and optimum frequency $(380 \mathrm{~Hz})$

\subsection{Effects of taped natural female mosquito sound (FM) on the male.}

Figure 4. shows the effect of the natural sound on the Anopheles gambiae males. The number attracted to the source was a function of distance. The longest distance $(2 \mathrm{~m}$ ) between the source and the test insects and the shortest distance of $0.5 \mathrm{~m}$. were found to be distinctly different from each other in terms of attraction. The shortest distance being the most attractive.

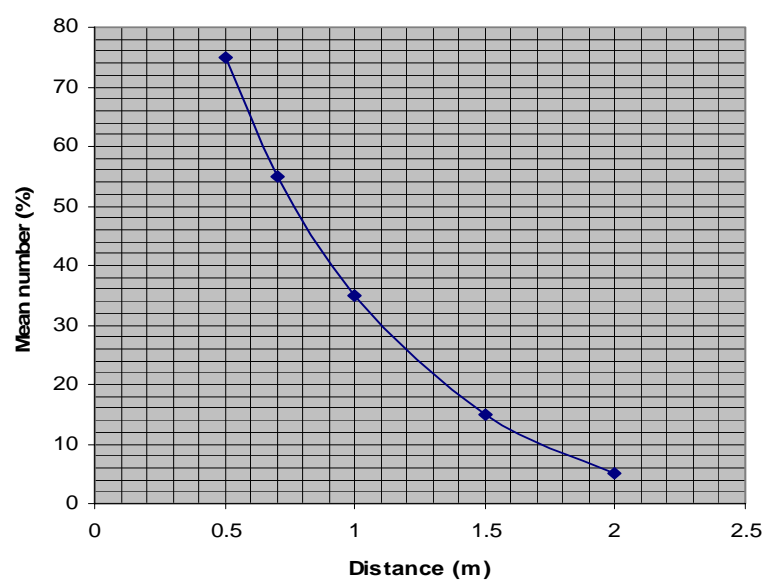

Figure 4. Males response to taped FM waves at different distances

\subsection{Effect of the PWM and FM broadcast in a choice test on the male mosquito}

Results on the choice between PWM and FM indicated that the male mosquitoes were attracted more by the PWM source than the FM source. As the optimum distance $(0.5 \mathrm{~m})$, was approached, the number towards the PWM increased as the number towards the PM source reduced proportionally (Figure 5). At same distance, $10 \%$ of the total number were attracted equally by both sources and made oscillatory motion between the two sources, hence termed as the 'undecided, group.

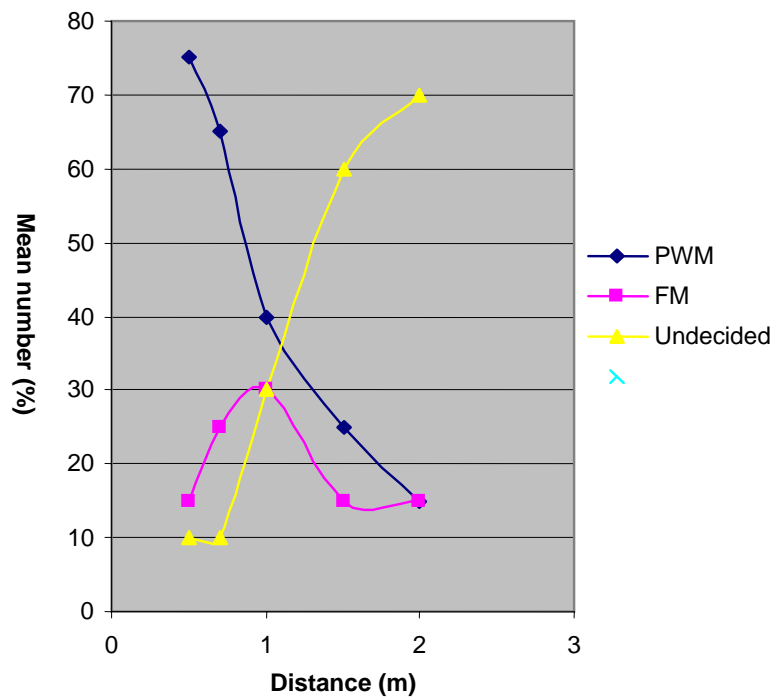

Figure 5. Two choice comparison of male mosquito's Response to PWM and FM sources

\subsection{Comparative effect of PWM and FM broadcasts on non-flying male mosquitoes}

In a search for measurable behaviour response to PWM broadcast, male Anopheles gambiae mosquitoes were observed for twenty minutes inside 
the net after the broadcast was switched off. They were allowed to settle in different parts of the net with some remaining within the vicinity of the speaker. The PWM source was then switched on and the mosquito response noted (Figure6) and scored as in Section 2.3.

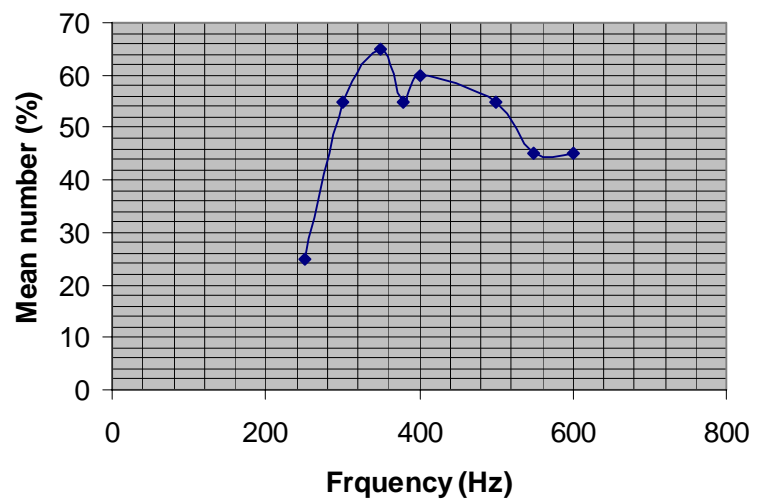

Figure 6. Effect of PWM broadcast on on quiescent males at optimum distance $(0.5 \mathrm{~m})$

\section{Discussion and Conclusion}

A wing-fanning insect (or a broadcast speaker) produces mechanical vibrations in air that can be detected over short distances by vibration sensors on the antenna of many insects. Male of swarming species of chironomids use their auditory system (Johnston's organ) to recognize a female within the swarm ${ }^{9}$. The role of acoustic behaviour in this phenomenon in Anopheles gambiae mosquitoes showed a similar trend. The results showed that the male Johnston's organ is sensitive to female flight tones from a distance of about $0.5 \mathrm{~m}-1.5 \mathrm{~m}$. The maximum approach rate at each frequency was at a distance of 0.5 meters, which is the radius of a sphere enclosing the electrically small antenna of the male mosquito. This distance gives the lowest value of the quality factor, $\mathrm{Q}$ of the antenna and is equal to the wave length of the radiated wave ${ }^{5}$. It gives the range of the antenna for acoustic communication in the radiative near field zone. The choice bioassay is conclusive evidence that a PWM source is more attractive to males than the natural sound mimics of the females. When given a choice, the males prefer a PWM to the natural FM emitted by the female mosquitoes, this is because of the superior mode of transmission of the PWM waves. Again, the PWM transmitter's frequency ranges can be varied from a low of $100 \mathrm{~Hz}$ to a high of a few kilohertz, unlike the FM waves emitted by the female. Since the PWM transmitter's frequency can be varied over a range of frequencies, the PWM serves duo purposes. An increased duration of stay of the male in the presence of a broadcast, is consistent with the observation that a female Mediterranean fruit fly often stops walking and remains close to a male ${ }^{10)}$ or turns to follow the male ${ }^{11)}$. We suspect that after contact, other cues, such as chemotactile takes over the communication and recognition becomes definite. This explains why the numbers that have contacted the PWM do not stay at the vicinity. A study of the response of the male mosquitoes to the audio frequency source shows that frequencies in the range 250-621 Hz elicit an attractive and repulsive response in male mosquitoes. The maximum attractive response occurs at $380 \mathrm{~Hz}$ and is most effective at short ranges between $0.5 \mathrm{~m}$ and $2 \mathrm{~m}$. The small attractive range can be attributed to the fact that the communication in these insects is a radiative near-field one, which is entirely dependent on the physical structure of the male's antennae.

\section{References}

1. A.N. Clement, Biology of Mosquitoes, Chapman and Hall, U.K., 1992.

2. A.W. Ewing, Anthropod Bioacouctics, Edinburgh University Press, U.K. 62, 1989.

3. P.L. Lane and R.W. Crosskey, Medical Insects and Arachnids, Chapman and Hall, U.K., 1996.

4. G. Baker, Magnetic Influence on the Activity of Termites and their Directional Behavior in Building Vertical Galleries, Mater.Org. 14, 534548, 1979.

5. S.J. Mclean, A re-examination of the fundamental limits on the radiation Q of electrically small antenna. IEEE Transaction Antennas and Propagation, 16:5, 619-671, 1996.

6. K. Gupta, Hand Book of Electronics, Pragati and prakash publishers, India, 1445-1475, 1996.

7. T.J. Walker, Sound Traps for Sampling Mole Cricket Flights (Orthoptera: Cryllotalpidae: Scapteriscus), Fla. Entomo, 65, 105-110, 1982.

8. O.M. Maweu, L.M. Muia, and A.L. Deng., Transmission Parameters of an Audio-rate Frequency Modulated Sound of a Female Mosquito, Asia J. of Phys, (accepted) (2009).

9. M.V. Fyodorova and A.I. Azovsky, (2003). Interaction Between Swarming Chironomid Annularius (Diptera:chironomdae) Males: Role of Acoustic Behavior. Journal of Insects, 16:2, 295 - 306, 2003.

10. Liimatainen, J. A. Hoikkala and T. Shelly, Courtship Behaviour in Ceratitis Capitata (Diptera:Tephritidae), Ann. Entomol.Soc. Am., 98, 1301-1308, 1977.

11. R.D. Briceno, Decisions During Courtship by Male and Female Medflies (Diptera, Tephritidae): Correlated Changes in the Male Behavior and Female Acceptance Criteria in Mass-reared Med Flies. Fla. Entomol, 85, 14-31, 2002. 